\title{
A new method to measure the semantic similarity from query phenotypic abnormalities to diseases based on the human phenotype ontology
}

Xiaofeng Gong, Jianping Jiang, Zhongqu Duan and Hui Lu*

From The Sixteenth Asia Pacific Bioinformatics Conference

Yokohama, Japan. 15-17 January 2018

\begin{abstract}
Background: Although rapid developed sequencing technologies make it possible for genotype data to be used in clinical diagnosis, it is still challenging for clinicians to understand the results of sequencing and make correct judgement based on them. Before this, diagnosis based on clinical features held a leading position. With the establishment of the Human Phenotype Ontology (HPO) and the enrichment of phenotype-disease annotations, there throws much more attention to the improvement of phenotype-based diagnosis.

Results: In this study, we presented a novel method called RelativeBestPair to measure similarity from the query terms to hereditary diseases based on HPO and then rank the candidate diseases. To evaluate the performance, we simulated a set of patients based on 44 complex diseases. Besides, by adding noise or imprecision or both, cases closer to real clinical conditions were generated. Thus, four simulated datasets were used to make comparison among RelativeBestPair and seven existing semantic similarity measures. RelativeBestPair ranked the underlying disease as top 1 on $93.73 \%$ of the simulated dataset without noise and imprecision, $93.64 \%$ of the simulated dataset with noise and without imprecision, $39.82 \%$ of the simulated dataset without noise and with imprecision, and $33.64 \%$ of the simulated dataset with both noise and imprecision.

Conclusion: Compared with the seven existing semantic similarity measures, RelativeBestPair showed similar performance in two datasets without imprecision. While RelativeBestPair appeared to be equal to Resnik and better than other six methods in the simulated dataset without noise and with imprecision, it significantly outperformed all other seven methods in the simulated dataset with both noise and imprecision. It can be indicated that RelativeBestPair might be of great help in clinical setting.
\end{abstract}

Keywords: Human phenotype ontology (HPO), Semantic similarity, Disease, Diagnosis

\footnotetext{
* Correspondence: huilu@sjtu.edu.cn

Department of Bioinformatics and Biostatistics, SJTU-Yale Joint Center for

Biostatistics, Shanghai Jiao Tong University, Shanghai, China
} 


\section{Background}

Correct diagnosis based on the observed clinical features of patients is a quite important task for physicians, especially in the field of rare genetic diseases, where different diseases often share some features. Recently, with the rapid development of sequencing technology, it becomes possible to improve diagnosis by providing physicians with patients' genotype data in a short time [1]. While techniques like whole genome sequencing and whole exome sequencing allows a patient's genotype data to be used to detect mutations, the relative high expense and the ability to identify disease-causing variants make it difficult to be put into practical clinical use. However, back to the beginning, if the performance of diagnosis based on clinical features can be improved, it will be of great help to the clinicians.

Thus, to make full use of clinical features or phenotypic information, many databases have been established to record and reorganize phenotypic data of diseases, such as OMIM [2] and Orphanet [3]. Furthermore, the Human Phenotype Ontology (HPO) [4-6] was constructed to describe human phenotype abnormalities in a structured and controlled vocabulary and has been widely used in research.

Recently, HPO has been widely applied in various fields. A web application called the Phenimizer provides ontology similarity search based on HPO to assist the clinical diagnosis workflow [7]. PhenoTips, a deep phenotyping tool and database, is developed to collect phenotypic information of patients with genetic disorders using HPO and suggest additional clinical investigations and possible disorders in Online Mendelian Inheritance in Man (OMIM) [8]. PhenoDB, a Web-based portal which can store and analyze phenotypic information using mapped HPO terms as well as other clinical information, is also developed [9]. Besides, several methods or tools have been introduced to combine phenotypic information based on HPO and genotypic data with other information available to make variant or gene prioritization, including eXtasy [10], Phen-Gen [11], an initial study using semantic similarity [12], PHIVE/Exomiser [13], Phevor [14], PhenoVar [15], PhenIx [16] and OMIM Explorer [17]. Despite the short history of HPO, it has drawn much attention from researchers and scientists and been broadly used in scientific researches.

In this article, we focus on using similarity between observed phenotypes of a patient and the annotated phenotypes of diseases to rank the candidate diseases of the patient. From this point of view, several methods and tools $[7,12,18]$ has been presented to exploit HPObased semantic similarity borrowing ideas from semantic similarity measures used in Gene Ontology (GO), which have been widely studied and broadly used during the last decade. Most of them utilized information content (IC) to calculate the semantic similarity. Although those approaches have been used in clinical research, the results are still uncertain and can be further imporved. Here we present a new method called RelativeBestPair. RelativeBestPair takes the ideas from information content and the best pair method. Our work shows better diagnosis using the RelativeBestPair method over other methods.

\section{Methods}

\section{Human phenotype ontology (HPO)}

An ontology is a knowledge-based structured system, which consists of a rich, standardized vocabulary to describe entities and the semantic relationships between them. The Human Phenotype Ontology (HPO) provides a standardized vocabulary of phenotypic abnormalities encountered in human disease. Terms in HPO, representing different phenotypic abnormalities, are related to their parent terms by "is a" relationship in a relaxed hierarchy which allows a term to possibly have multiple parent terms (Fig. 1). With HPO terms corresponding to phenotypic abnormalities, diseases can be described in a detailed and organized way. The HPO (version 1.2 releases/2017$2-14)$ currently contains approximately 12,000 terms (still growing) and over 120,000 phenotype-disease annotations. Here we concentrate on annotations about 6918 diseases listed in Online Mendelian Inheritance in Man (OMIM) to calculate the semantic similarity scores.

\section{RelativeBestPair method}

Based on the HPO structure and annotations, the information content of a term $t$ in HPO is defined as follows:

$$
\mathrm{IC}(t)=-\log \frac{N_{t}}{N}
$$

where $N$ is the total number of annotated diseases and $N_{t}$ is the number of diseases annotated by term $t$ and all its descendants. When comparing the similarity between two sets of phenotypes, the best pair method just simply counts the number of same terms in both two sets, which does not take the semantic inheritance structure of HPO and the different importance of the terms into consideration.

Thus we propose RelativeBestPair, a new semantic similarity measure based on the information content and the best pair method. Inspired by the idea of information content, we collect diseases annotated by a phenotype $t$ and its descendants to measure the different importance of terms. RealtiveBestPair is described as follows.

A. For a given term $t$, we denote $D(t)$ as the set of diseases annotated by term $t$ and all its descendants and $N_{t}$ as the size of $D(t)$. Then, the sccn term $t$ is defined as 


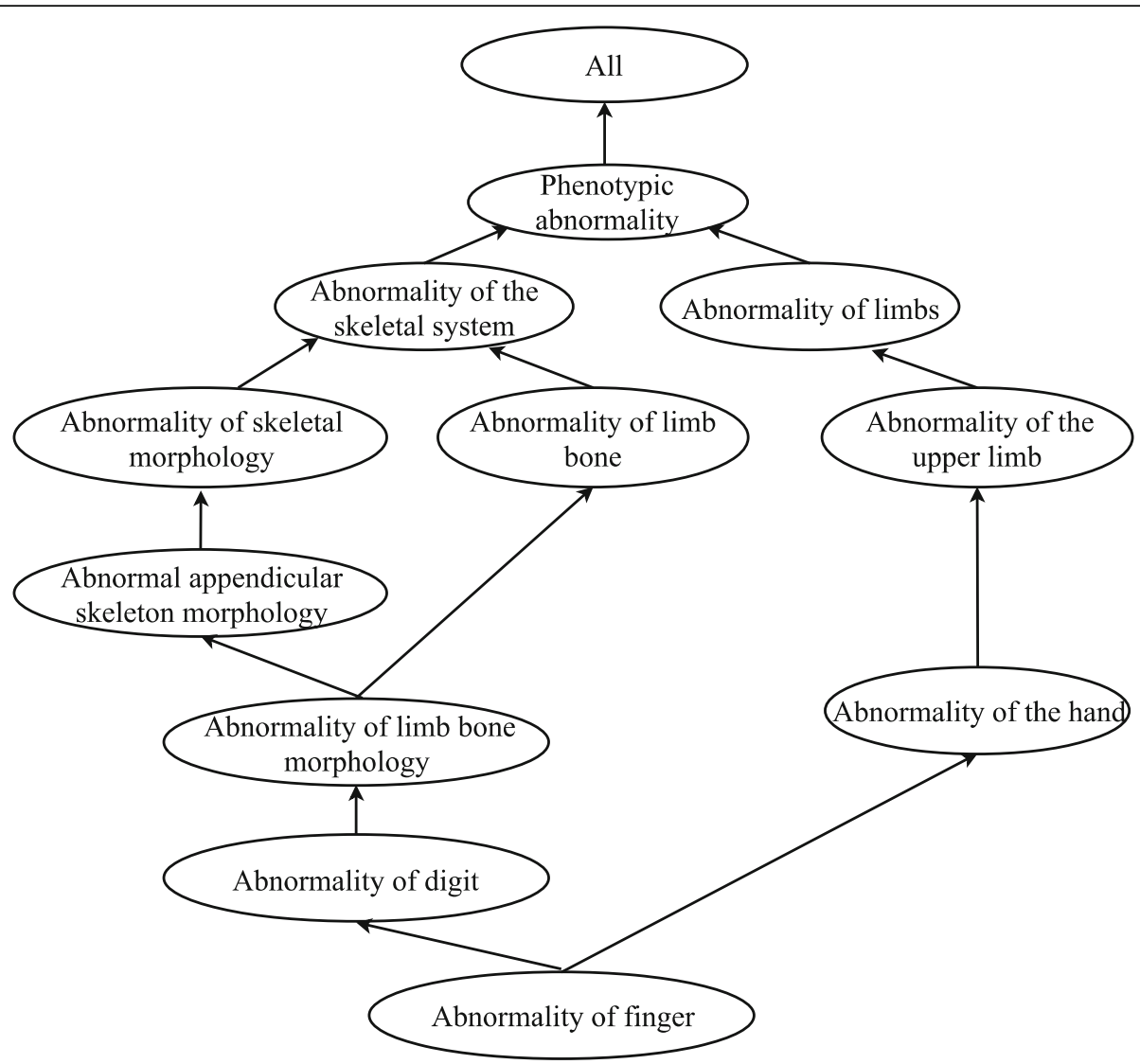

Fig. 1 Example of the structure of HPO. Term Abnormality of finger (HP:0001167) and all its ancestors are shown. Each term, representing a phenotypic abnormality, is related to parents terms by "is a" relationship

$$
S(D \mid t)=\left\{\begin{array}{cc}
1 / N_{t}, & D \in d(t) \\
0, & \text { otherwise }
\end{array}\right.
$$

B. Then we can get all the scores of being each disease given each term. For a sets of phenotypes $\left\{t_{1}, t_{2}, \ldots\right.$, $\left.t_{n}\right\}$ and a disease $\mathrm{D}_{k}$, the semantic similarity score can be calculated as

$$
\operatorname{Sim}\left(D_{k} \mid t_{1}, t_{2}, \ldots, t_{n}\right)=\sum_{i=1}^{n} \min \left(\alpha, S\left(D_{k} \mid t_{i}\right)\right)
$$

where $\alpha$ is a given threshold.

The threshold $\alpha$ is introduced to control the contribution of a single term. If only several diseases are annotated by a single term, then the score of being one of those diseases given this term will be so large that it may dominate the semantic similarity score and ignore the contributions of other terms. For example, we observed a patient with ten terms $\left\{t_{1}, t_{2}, \ldots, t_{10}\right\}$. If the score of being $\mathrm{D}_{1}$ given each of $\left\{t_{1}, t_{2}, \ldots, t_{9}\right\}$ is suitable like 0.005 while the score of being $\mathrm{D}_{2}$ given $t_{10}$ is quite large, for example 0.1 , the semantic similarity score between the patient and $D_{2}$ will be larger than that between the patient and $D_{1}$. Thus we use the threshold $\alpha$ to avoid the such extreme cases. Although the choice of $\alpha$ may affect the performance, generally we set it to be 0.01 .

Disease diagnosis based on RelaitveBestPair can be summarized as followed (Fig. 2). With the input of HPO and its annotations, the ontology and the database (containing the scores of being each Disease D given each term t using Eq. (2)) are constructed first. Then given a query set of phenotype terms, the similarity scores from query terms to each disease can be calculated with Eq. (3). Finally, diseases are ranked according to these scores from the largest to the smallest.

\section{Existing semantic similarity measures}

We compared the performance of RelativeBestPair with other seven existing approaches summarized in HPOsim [19]. Among those, six approaches are based on information content. The Resnik measure [20], the Lin measure [21], the Jiang-Conrath measure [22], the Relevance measure [23], the information coefficient measure [24] and the graph IC measure [25] define the similarity between two terms as follows:

$$
\operatorname{sim}_{\text {Resnik }}\left(t_{1}, t_{2}\right)=I C\left(t_{M I C A}\right)
$$




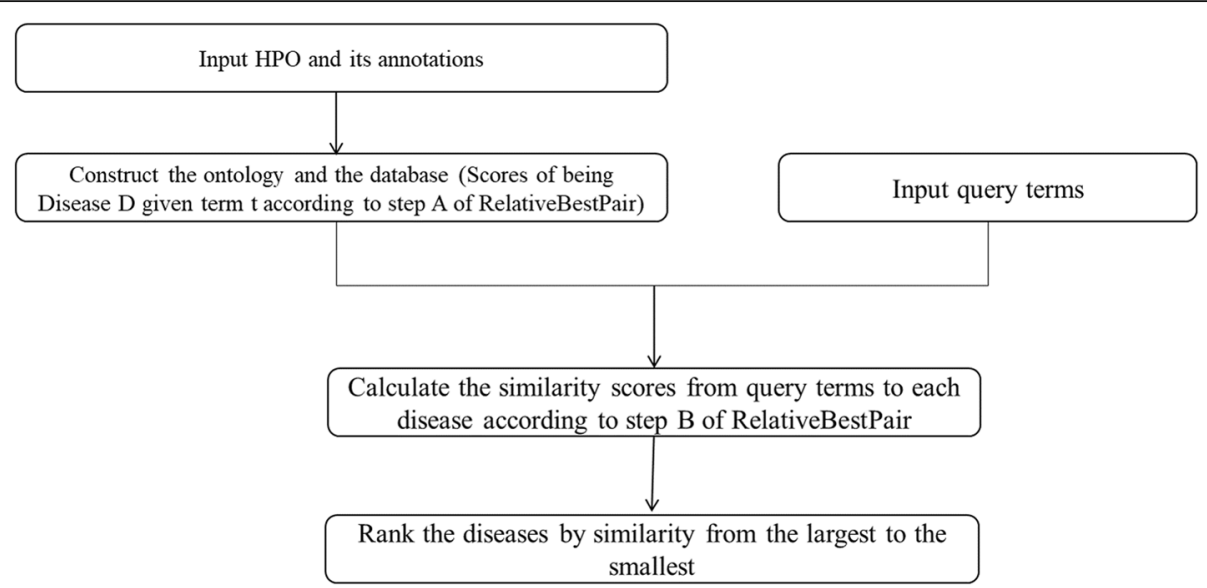

Fig. 2 The workflow of disease diagnosis based on RelativeBestPair

$$
\begin{aligned}
& \operatorname{sim}_{\text {Lin }}\left(t_{1}, t_{2}\right)=\frac{2 \times I C\left(t_{M I C A}\right)}{I C\left(t_{1}\right)+I C\left(t_{2}\right)} \\
& \operatorname{sim}_{J C}\left(t_{1}, t_{2}\right)=\frac{1}{\left(1+I C\left(t_{1}\right)+I C\left(t_{2}\right)-2 \times I C\left(t_{M I C A}\right)\right)} \\
& \operatorname{sim}_{\text {Rel }}\left(t_{1}, t_{2}\right)=\operatorname{sim}_{L i n}\left(t_{1}, t_{2}\right) \times\left(1-p\left(t_{M I C A}\right)\right) \\
& \operatorname{sim}_{I C}\left(t_{1}, t_{2}\right)=\operatorname{sim}_{L i n}\left(t_{1}, t_{2}\right) \times\left(1-\frac{1}{1+I C\left(t_{M I C A}\right)}\right) \\
& \operatorname{sim}_{\text {GraphIC }}\left(t_{1}, t_{2}\right)=\frac{\sum_{t \in\left(A\left(t_{1}\right) \cap A\left(t_{2}\right)\right)} I C(t)}{\sum_{t \in\left(A\left(t_{1}\right) \cap A\left(t_{2}\right)\right)} I C(t)}
\end{aligned}
$$

Where IC is defined as (1), $t_{M I C A}$ is the most informative common ancestors, $p\left(t_{M I C A}\right)$ is the proportion of diseases annotated by $t_{M I C A}$ and $A(t)$ is the set of the ancestors of term $t$ in HPO.

Besides, the Wang measure [26] is based the structure of ontology. For a given term $t, D A G_{t}=\left(t, T_{t}, E_{t}\right)$ represents the subgraph made up of term $t$ and its ancestors, where $T_{t}$ is the set of the ancestors of $t$ and $E_{t}$ is the corresponding set of edges is $D A G_{t}$. In $D A G_{t}, S_{t}(n)$ is defined as:

$$
\left\{\begin{array}{c}
S_{t}(t)=1 \\
S_{t}(n)=\max \left\{w_{e} * S_{t}\left(n^{\prime}\right) \mid n^{\prime} \in \text { children of }(n)\right\} \text { if } \mathrm{t} \neq \mathrm{n}
\end{array}\right.
$$

here we choose $w_{e}$ equal to 0.8 . Therefore the similarity between two terms is defined as:



Fig. 3 The workflow of disease diagnosis based on the seven existing methods 


$$
\operatorname{sim}_{\text {Wang }}\left(t_{1}, t_{2}\right)=\frac{\sum_{t \in T_{t 1} \cap T t_{2}} S_{t_{1}}(t)+S_{t_{2}}(t)}{S V\left(t_{1}\right)+S V\left(t_{2}\right)}
$$

where $S V(t)$ is the sum of $S_{t}(n)$ for $n$ in $D A G_{t}$.

In order to get the similarity between the query set of terms and the set of disease associated terms, we used the one-sided search algorithm as it was showed to be superior to the symmetric version in [7]. The one-sided search algorithm is defined as:

$$
\operatorname{sim}_{\text {one-sided }}(Q \rightarrow D)=\operatorname{avg}\left[\sum_{t_{1} \in Q} \max _{t_{2} \in D} \operatorname{sim}\left(t_{1}, t_{2}\right)\right]
$$

where $Q$ is the set of the query terms (observed phenotypes of the patient), $D$ is the set of terms annotated with a given disease, and $\operatorname{sim}\left(t_{1}, t_{2}\right)$ can be one of the seven approaches.

Disease diagnosis based the seven semantic similarity measures is quite similar with that based on.

RelativeBestPair (Fig. 3). Firstly, the ontology and the database (containing information content of each term in HPO) are constructed based on HPO and its annotation files. Secondly, given a query set of phenotype terms, the similarity score from these query terms to each disease are calculated with term-term similarity based on each of the seven methods and then one-sided search algorithm. Finally, diseases are also ranked from the largest score to the smallest score.

\section{Performance evaluation and generation of simulated patients}

Since it is difficult to get clinical features about a large number of patients, we used similar method and same data in [7] to generate simulated patients. In the data used in [7], 44 complex dysmorphology syndromes were identified with detailed frequency of phenotypes. The simulation process is as follows. First, we assigned a disease to each patient. Second, for each phenotype associated with the assigned disease, a random integer between 0 and 100 was generated. If the number was smaller than the relative occurrence in 100 patients (frequency"100), the corresponding phenotype was kept. For each of the 44 diseases, we generated 25 patients with at least three phenotypes. Finally, we got a dataset of 1100 simulated patients. To make the simulation more realistic, three more datasets were also generated just as what was done in $[7,12]$. We generated a dataset with 'noise' by adding half as many noise terms, unrelated with the underlying disorder, to the present terms, a dataset with 'imprecision' by randomly substituting each of the present phenotypes with one of its ancestors in HPO, and also a dataset with both 'imprecision' and 'noise' by imprecision step first and then noise step. With the four simulated datasets, we evaluated the performance of semantic
Table 1 Summary results of different methods on the four

\begin{tabular}{|c|c|c|c|c|c|c|c|c|}
\hline \multicolumn{9}{|c|}{ Dataset 1(Noise:-, Imprecision:-) } \\
\hline & Resnik & Lin & $J C$ & Rel & IC & GraphIC & Wang & RBP \\
\hline Top 1 & 1027 & 1016 & 1029 & 1018 & 1021 & 1029 & 1023 & 1031 \\
\hline Top 5 & 1087 & 1071 & 1082 & 1071 & 1075 & 1079 & 1078 & 1091 \\
\hline Top 10 & 1089 & 1077 & 1088 & 1077 & 1079 & 1081 & 1081 & 1095 \\
\hline op 20 & 1092 & 1078 & 1092 & 1078 & 1080 & 1083 & 1081 & 1096 \\
\hline \multicolumn{9}{|c|}{ Dataset 2(Noise:+, Imprecision:-) } \\
\hline & Resnik & Lin & $J C$ & Rel & IC & GraphIC & Wang & RBP \\
\hline op 1 & 992 & 997 & 1036 & 996 & 1006 & 1031 & 1001 & 1030 \\
\hline Top 5 & 1074 & 1059 & 1081 & 1063 & 1070 & 1077 & 1071 & 1089 \\
\hline$p$ p 10 & 1081 & 1069 & 1086 & 1071 & 1077 & 1080 & 1078 & 1094 \\
\hline Top 20 & 1087 & 1074 & 1089 & 1076 & 1078 & 1083 & 1079 & 1095 \\
\hline \multicolumn{9}{|c|}{ Dataset 3(Noise:-, Imprecision:+) } \\
\hline & Resnik & Lin & $J C$ & Rel & IC & GraphlC & Wang & RBP \\
\hline$p$ p 1 & 434 & 243 & 104 & 302 & 336 & 120 & 172 & 438 \\
\hline Top 5 & 767 & 502 & 261 & 583 & 603 & 341 & 446 & 765 \\
\hline$p$ p 10 & 866 & 613 & 342 & 685 & 707 & 482 & 602 & 863 \\
\hline Top 20 & 926 & 714 & 440 & 785 & 797 & 620 & 725 & 926 \\
\hline \multicolumn{9}{|c|}{ Dataset 4(Noise:+, Imprecision:+) } \\
\hline & Resnik & Lin & $J C$ & Rel & IC & GraphIC & Wang & RBP \\
\hline Top 1 & 183 & 130 & 97 & 143 & 162 & 73 & 77 & 370 \\
\hline Top 5 & 453 & 327 & 239 & 383 & 406 & 252 & 263 & 694 \\
\hline Top 10 & 579 & 452 & 319 & 509 & 533 & 393 & 384 & 786 \\
\hline op 20 & 703 & 570 & 420 & 640 & 657 & 540 & 535 & 860 \\
\hline
\end{tabular}
simulated datasets

Resnik the Resnik measure, Lin the Lin measure, $J C$ the Jiang-Conrath measure, Rel the Relevance measure, IC the information coefficient measure, Graph/C the graph IC measure, Wang the Wang measure, RBP RelativeBestPair method The seven existing measures are all implemented with one-sided search algorithm. The numbers represent the number of patients in 1100 cases that the true diseases are ranked within top 1, top 5, top 10 or top 20

similarity measure by the ranks of the true disease and adopted the criterion from $[12,19]$.

\section{Results}

We evaluated the performance of the seven existing approaches and RealtiveBestPair method in the four simulated datasets respectively. We denoted the dataset without noise and imprecision, the dataset with noise and without imprecision, the dataset without noise and with imprecision, and the dataset with both noise and imprecision as "Dataset 1(Noise:-, Imprecision:-)", "Dataset 2(Noise:+, Imprecision:)", "Dataset 3(Noise:-, Imprecision:+)", and "Dataset 4(Noise:+, Imprecision:+)". As we moved on from Dataset 1 to Dataset 4, it became more difficult to make the correct diagnosis. It would show us the real abilities of those methods to identify the true underlying disease.

For a given patient, we calculated the similarity score from the patient to each of the 6918 OMIM diseases using one kind of semantic similarity measure, and then 
rank all the diseases by their similarity scores (from the largest to the smallest). In case that some diseases received the same score, the average rank was returned to make it more reasonable. The results of all the eight methods on the four datasets are shown in Table 1 and Figs. 1, 2, 3 and 4.

It can be seen that in the seven existing semantic similarity measures, the Resnik measure has a modest advantage over other six approaches, similar to the results in [7]. The RelativeBestPair method shows the almost the best performance in all four datasets (Table 1). Although in Dataset 1 and Dataset 2, two datasets that do not include "imprecision", all methods reveal good results by ranking the true diseases as top 1 on over $90 \%$ of the patients and within top 20 on over $95 \%$ of the patients (Table 1, Figs. 4 and 5), their performances deteriorate with different extents in Dataset 3 and Dataset 4. In Dataset 3 with imprecision, RelativeBestPair method, along with the Resnik measure, tends to be superior with the underlying diseases being ranked within top 1, top 5, top 10 , top 20 on $39.82 \%, 69.55 \%, 78.45 \%, 84.18 \%$ of the cases for RealtiveBestPair and 39.45\%, 69.73\%, 78.73\%, $84.18 \%$ for Resnik (Table 1, Fig. 6). The corresponding percentages using other measures are much smaller. In Dataset 4 , a more real situation by both introducing unrelated phenotypic noise and using terms that are more general, RelativeBestPair achieves the best performance among the eight methods (Table 1, Fig. 7). On $33.64 \%$ of the patients, their underlying diseases are ranked the highest when applying RelativeBestPair. In comparison, the percentages using Resnik, Lin, Jiang-
Conrath, Relevance, information coefficients, Graph IC and Wang measures are only $16.64 \%, 11.82 \%, 8.82 \%$, $13 \%, 14.73 \%, 6.64 \%$ and $7 \%$ respectively. Even if a higher rank threshold is employed to give out a candidate list, RelativeBestPair still turns out to be significant better than other methods (Fig. 4). In total, it indicates that RelativeBestPair has the potential to provide a candidate disease/disease list for clinician to improve the diagnosis efficiency as well as accuracy.

\section{Discussion and conclusion}

Recently, the rapid development of sequencing technology makes it possible to get personal genotype data for clinical use, which may be helpful in disease diagnosis. However, the relative high cost and low ability to identify the disease-related causal variants prevent it from being widely used in real cases. While lots of effort and money have been paid to study the relationship between diseases and genetic mutations, to speed up the process of sequencing and to promote the accuracy of sequencing results, in this article we focus on the improvement in the field of phenotypic diagnosis. Compared with genotypic data, it is much easier to get phenotypic data from patients. With the construction and development of the Human Phenotype Ontology and the enrichment and completeness of disease-phenotype annotations, the observed phenotypes of a particular patient can provide more information about the underlying disease he/she might suffer.

Here we proposed a novel method called RelativeBestPair to measure the semantic similarity from a given set

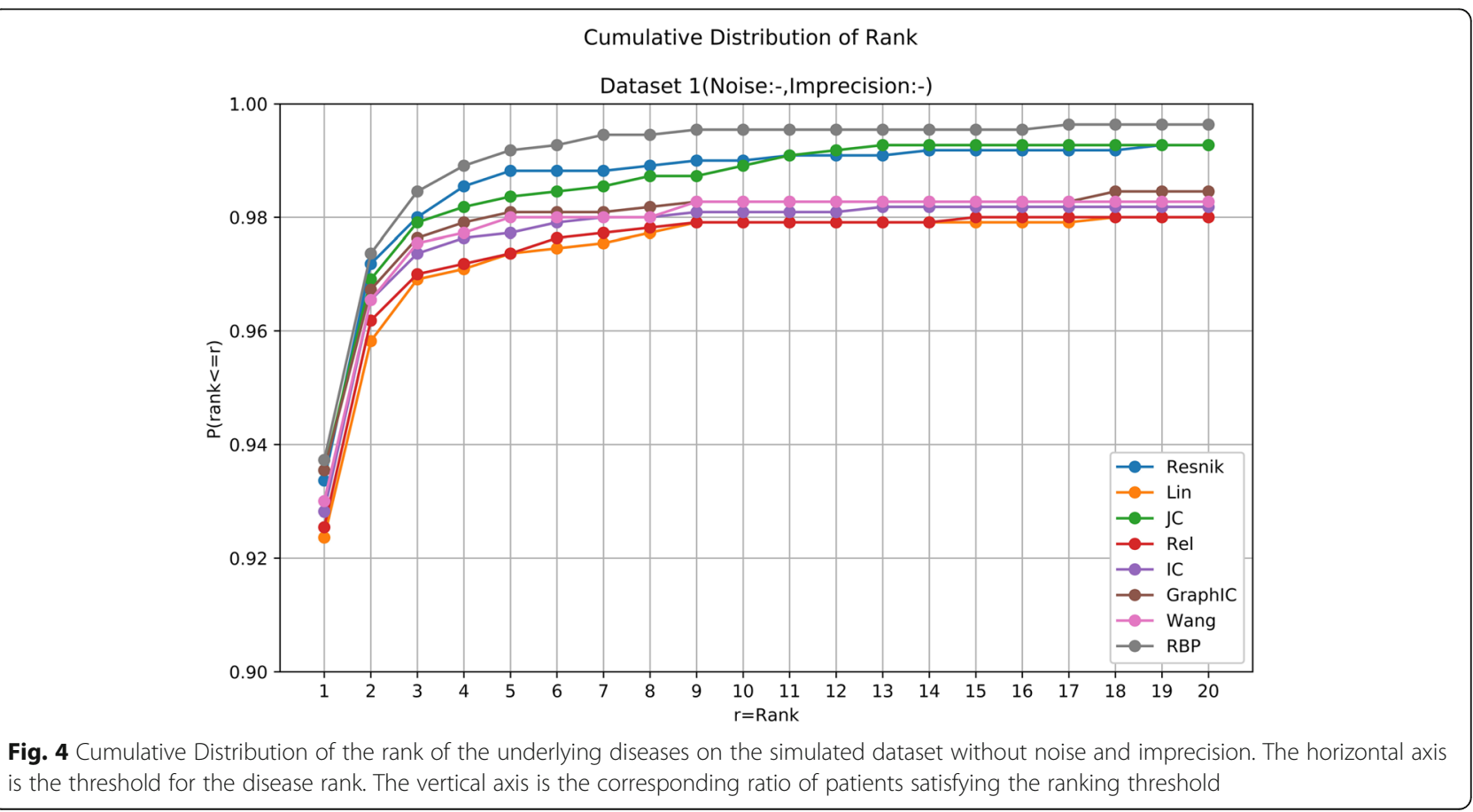




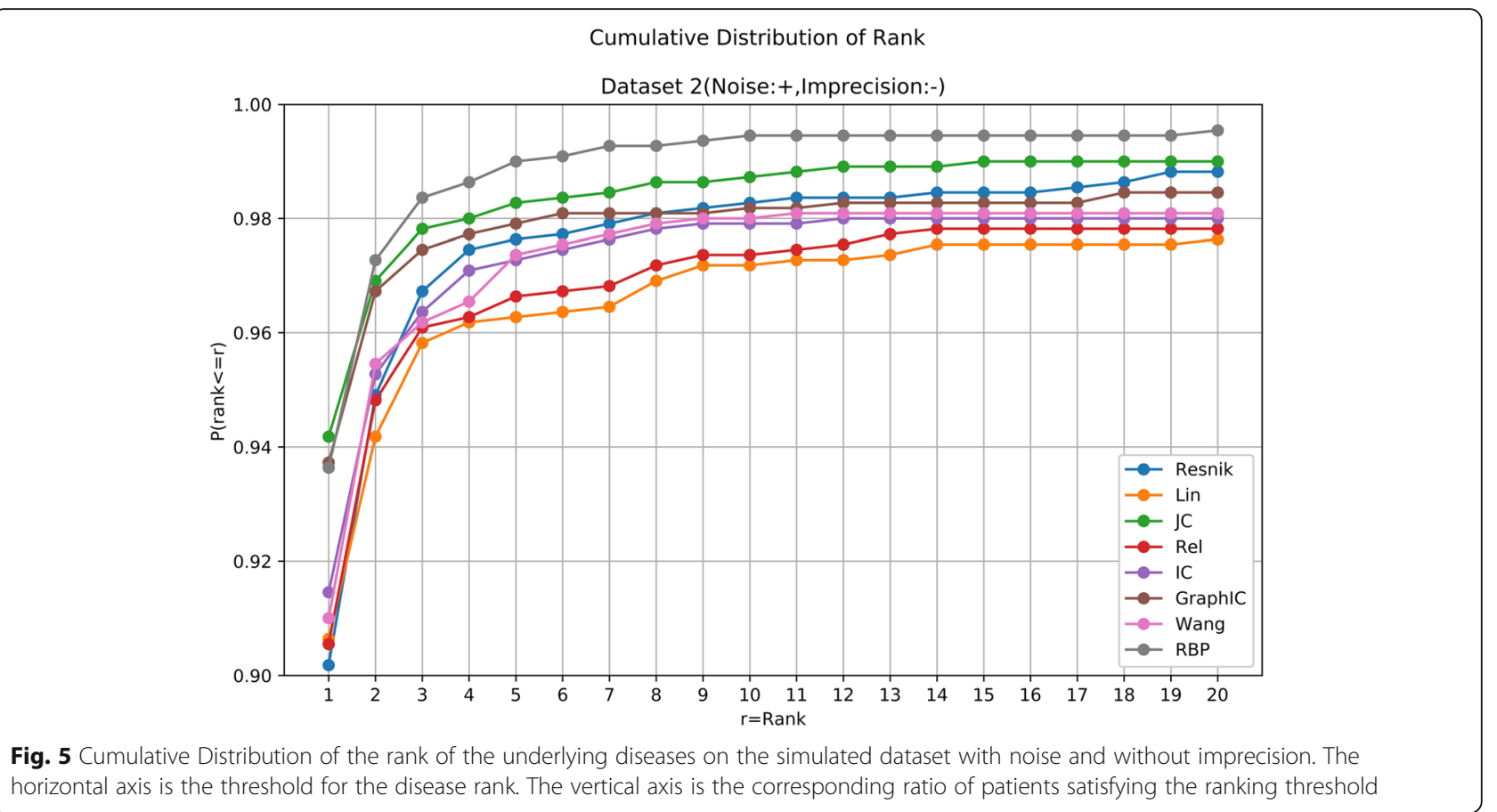

of phenotypes to a disease. Different from those existing approaches that calculate the similarity from the query set to a certain disease based on term-term comparison, we directly define the contribution of one phenotype term to the certain disease. To evaluate the performance of RelativeBestPair and seven existing methods, we adopted the procedure similar to that in $[7,12]$ to generate four kinds of simulated patients from the easiest situation to the most difficult situation. In order to be adapted to the scenario of disease diagnosis, the one-sided search algorithm, which showed better performance than symmetric version in [7], was chosen for the seven existing methods. The results on the simulated datasets demonstrated that RelativeBestPair outperformed other methods in all situations especially when "noise" and "imprecision" were added, typical in the clinical setting.

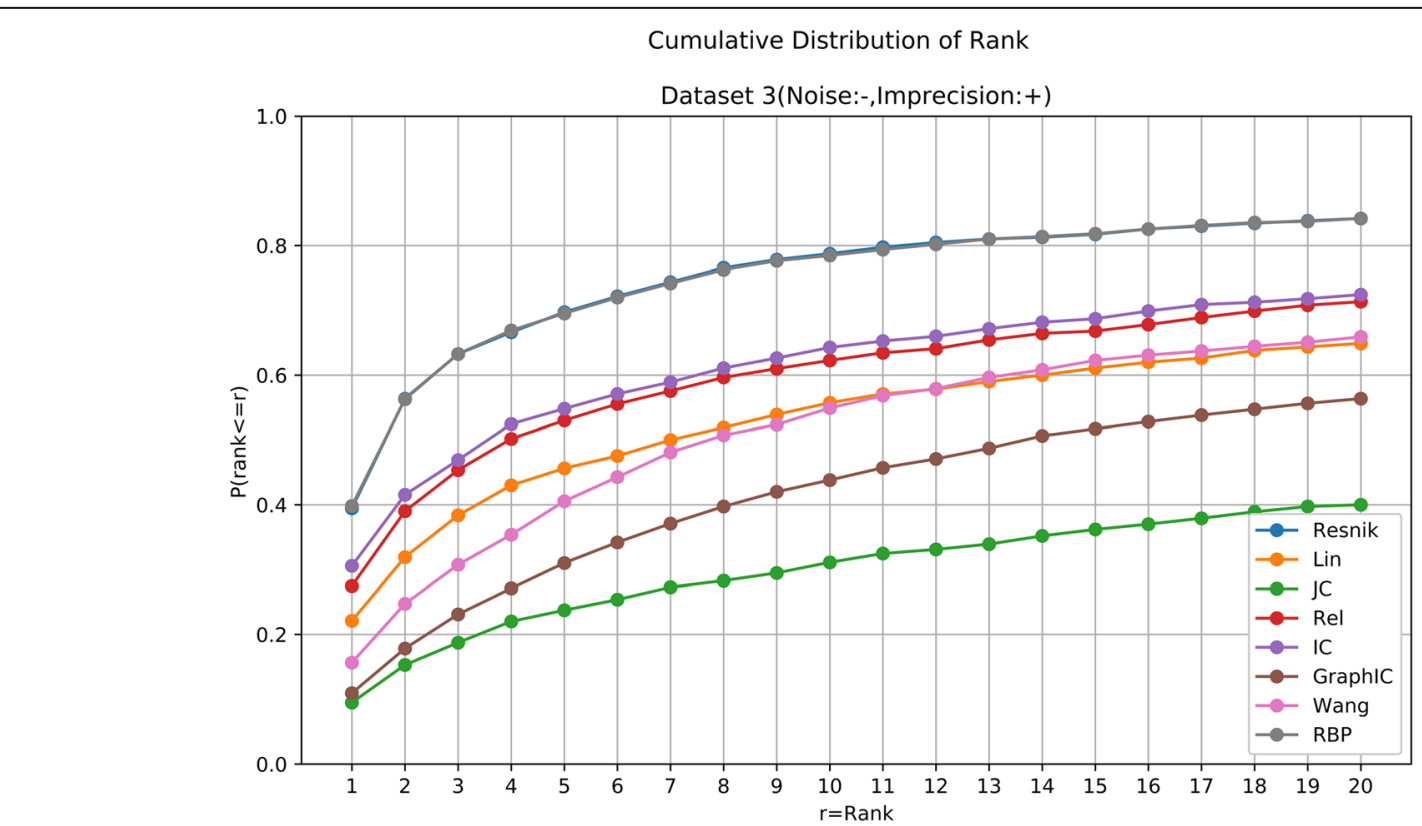

Fig. 6 Cumulative Distribution of the rank of the underlying diseases on the simulated dataset without noise and with imprecision. The horizontal axis is the threshold for the disease rank. The vertical axis is the corresponding ratio of patients satisfying the ranking threshold 


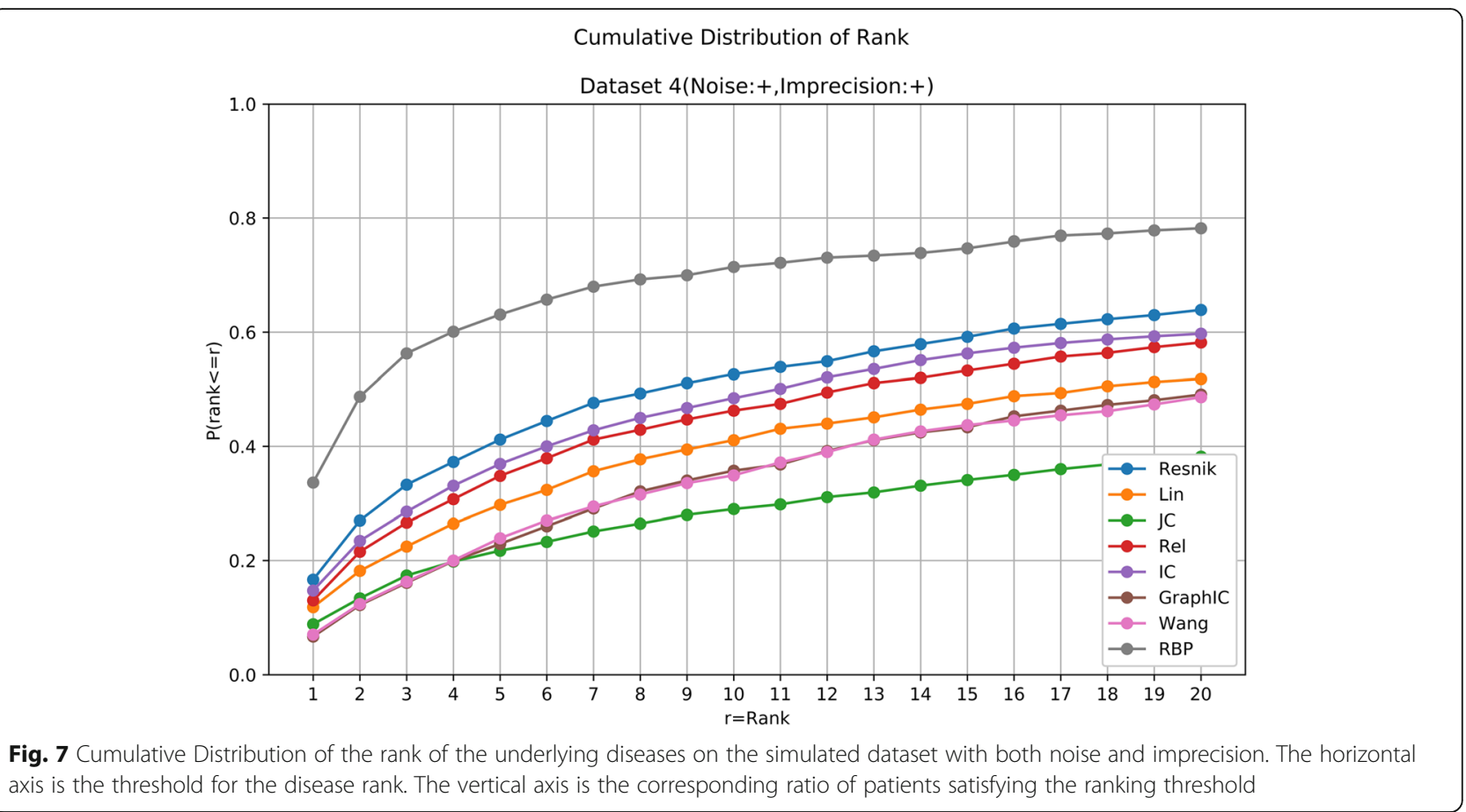

Despite the well performance in simulation, there still remains much for RelativeBestPair to take into consideration. Firstly, the optimal value for $\alpha$ requires further discussion. The introduction of threshold $\alpha$ played a key role in the performance of RelativeBestPair since we found poor results when the threshold $\alpha$ was not employed. Therefore, the choice of threshold $\alpha$ would substantially affect the performance. Other than 0.01 , we also tested other values for $\alpha$ including 0.001-0.005, $0.015,0.02,0.025$ and 0.03 . Although those results showed some minor difference (data not shown), considering the fact that on average one term annotates about 150 diseases which indicates that average score of being the given disease is $1 / 150 \approx 0.0067$, empirically the choice of 0.01 for $\alpha$ might be enough to make sure that the contribution of one single term won't be too large. Other choices are also welcomed as long as $\alpha$ is neither too big nor too small. Secondly, unlike the seven existing approaches, RelativeBestPair cannot be used to compute the similarity between two phenotype terms. The usage of RelativeBestPair might be limited in disease diagnosis and its expansion to other biomedical ontologies and other usages may be uncertain. Finally, without thousands of real cases, the true ability of RelativeBestPair as well as other semantic similarity measures in disease diagnosis is still unknown. As mentioned before, all the simulations are based on 44 complex diseases with detailed frequencies of phenotypes [7]. Then, we cannot assert the performance in any cases. However, from the simulation results, RelativeBestPair might have a large potential to identity the true underlying diseases of patients.
In conclusion, we have presented a new method, RelativeBestPair, that calculates the semantic similarity from the given query terms to each disease. Our method has the advantage of pay special attention to the fields of disease diagnosis. This approach can be applied to the real clinical setting by providing clinicians with a candidate disease list. We have shown that RelativeBestPair achieved a better performance of identifying the true disease as top-ranked diseases against other methods in four simulated dataset, mimic to the real cases.

\section{Acknowledgements}

We would like to thank Center for HPC, Shanghai Jiao Tong University and Children Hospital of Shanghai for providing computation help.

\section{Funding}

This work and the publication charges for this article was supported by the SJTU-Yale Seed Fund for Research collaborations and by the "Neil Shen's SJTU Medical Research Fund"

\section{Availability of data and materials}

The data used to generate simulated patients was reported by Köhler et al. in [7] and is available as supplemental data in http://www.cell.com/ajhg/ fulltext/S0002-9297(09)00399-1.

\section{About this supplement}

This article has been published as part of BMC Bioinformatics Volume 19 Supplement 4, 2018: Selected articles from the 16th Asia Pacific Bioinformatics Conference (APBC 2018): bioinformatics. The full contents of the supplement are available online at https://

bmcbioinformatics.biomedcentral.com/articles/supplements/volume-18supplement-17.

\section{Authors' contributions}

XFG designed and implemented the method. JPJ and ZQD provided valuable advice on the method. XFG prepared the data and analyzed the results. XFG wrote the manuscript. HL provided guidance and revised the manuscript. All authors read and approved the final manuscript. 
Ethics approval and consent to participate

Not applicable.

\section{Consent for publication}

Not applicable.

\section{Competing interests}

The authors declare that they have no competing interests.

\section{Publisher's Note}

Springer Nature remains neutral with regard to jurisdictional claims in published maps and institutional affiliations.

\section{Published: 8 May 2018}

\section{References}

1. Smedley D, Jacobsen JO, Jäger M, Köhler S, Holtgrewe M, Schubach M, et al. Next-generation diagnostics and disease-gene discovery with the exomiser. Nat Protoc. 2015;10(12):2004-2015.

2. Hamosh A, Scott AF, Amberger JS, Bocchini CA, McKusick VA. Online Mendelian inheritance in man (OMIM), a knowledgebase of human genes and genetic disorders. Nucleic Acids Res. 2005;33(suppl 1):D514-7.

3. Aymé S. Orphanet, an information site on rare diseases. Soins. 2003;672:46.

4. Robinson PN, Köhler S, Bauer S, Seelow D, Horn D, Mundlos S. The human phenotype ontology: a tool for annotating and analyzing human hereditary disease. Am J Hum Genet. 2008;83(5):610-5.

5. Köhler S, Doelken SC, Mungall CJ, Bauer S, Firth HV, Bailleul-Forestier I, et al. The human phenotype ontology project: linking molecular biology and disease through phenotype data. Nucleic Acids Res. 2014;42(D1):D966-74.

6. Köhler S, Vasilevsky NA, Engelstad M, Foster E, McMurry J, Aymé S, et al. The human phenotype ontology in 2017. Nucleic Acids Res. 2017; 45(D1):D865-76.

7. Köhler S, Schulz MH, Krawitz P, Bauer S, Dölken S, Ott CE, et al. Clinical diagnostics in human genetics with semantic similarity searches in ontologies. Am J Hum Genet. 2009;85(4):457-64.

8. Girdea M, Dumitriu S, Fiume M, Bowdin S, Boycott KM, Chénier S, et al. PhenoTips: patient phenotyping software for clinical and research use. Hum Mutat. 2013;34(8):1057-65.

9. Hamosh A, Sobreira N, Hoover-Fong J, Sutton VR, Boehm C, Schiettecatte F, Valle D. PhenoDB: a new web-based tool for the collection, storage, and analysis of phenotypic features. Hum Mutat. 2013:34(4):566-71.

10. Sifrim A, Popovic D, Tranchevent LC, Ardeshirdavani A, Sakai R, Konings P, et al. eXtasy: variant prioritization by genomic data fusion. Nat Methods. 2013; 10(11):1083-4.

11. Javed A, Agrawal S, Ng PC. Phen-gen: combining phenotype and genotype to analyze rare disorders. Nat Methods. 2014;11(9):935-7.

12. Masino AJ, Dechene ET, Dulik MC, Wilkens A, Spinner NB, Krantz ID, et al. Clinical phenotype-based gene prioritization: an initial study using semantic similarity and the human phenotype ontology. BMC Bioinform. 2014;15(1):248.

13. Robinson PN, Köhler S, Oellrich A, Wang K, Mungall CJ, Lewis SE, et al. Improved exome prioritization of disease genes through cross-species phenotype comparison. Genome Res. 2014;24(2):340-8.

14. Singleton MV, Guthery SL, Voelkerding KV, Chen K, Kennedy B, Margraf RL, et al. Phevor combines multiple biomedical ontologies for accurate identification of disease-causing alleles in single individuals and small nuclear families. Am J Hum Genet. 2014;94(4):599-610.

15. Trakadis YJ, Buote C, Therriault JF, Jacques PÉ, Larochelle H, Lévesque S. PhenoVar: a phenotype-driven approach in clinical genomics for the diagnosis of polymalformative syndromes. BMC Med Genet. 2014;7(1):22.

16. Zemojtel $T$, Köhler $S$, Mackenroth $L$, Jäger $M$, Hecht J, Krawitz $P$, et al. Effective diagnosis of genetic disease by computational phenotype analysis of the disease-associated genome. Sci Transl Med. 2014;6(252):252ra123252 ra123.

17. James RA, Campbell IM, Chen ES, Boone PM, Rao MA, Bainbridge MN, et al. A visual and curatorial approach to clinical variant prioritization and disease gene discovery in genome-wide diagnostics. Genome Med. 2016;8(1):13.

18. Deng Y, Gao L, Wang B, Guo X. HPOSim: an R package for phenotypic similarity measure and enrichment analysis based on the human phenotype ontology. PLoS One. 2015;10(2):e0115692.
19. Peng J, Xue H, Shao Y, Shang X, Wang Y, Chen J. Measuring phenotype semantic similarity using human phenotype ontology. In bioinformatics and biomedicine (BIBM), 2016 IEEE international conference on Shenzhen: IEEE; 2016. pp. 763-6.

20. Resnik P. Using information content to evaluate semantic similarity in a taxonomy. In: Proceedings of the 14th international joint conference on artificial intelligence (IJCAI-95); 1995.

21. Lin D. An information-theoretic definition of similarity. In: ICML, vol. Vol. 98 , no. 1998; 1998. p. 296-304.

22. Jiang JJ, Conrath DW. Semantic similarity based on corpus statistics and lexical taxonomy. In: Proc of 10th international conference on research in computational linguistics, ROCLING'97; 1997.

23. Schlicker A, Domingues FS, Rahnenführer J, Lengauer T. A new measure for functional similarity of gene products based on gene ontology. BMC Bioinforma. 2006:7(1):302.

24. Li, B., Wang, J. Z., Feltus, F. A., Zhou, J., \& Luo, F. (2010). Effectively integrating information content and structural relationship to improve the GO-based similarity measure between proteins. arXiv preprint arXiv: 1001.0958.

25. Pesquita C, Faria D, Bastos H, Falcao A, Couto F. Evaluating GO-based semantic similarity measures. In: Proc. 10th annual bio-ontologies meeting, vol. Vol. 37, no. 40; 2007. p. 38.

26. Wang JZ, Du Z, Payattakool R, Yu PS, Chen CF. A new method to measure the semantic similarity of GO terms. Bioinformatics. 2007:23(10):1274-81.

\section{Submit your next manuscript to BioMed Central and we will help you at every step:}

- We accept pre-submission inquiries

- Our selector tool helps you to find the most relevant journal

- We provide round the clock customer support

- Convenient online submission

- Thorough peer review

- Inclusion in PubMed and all major indexing services

- Maximum visibility for your research

Submit your manuscript at www.biomedcentral.com/submit
Biomed Central 